\title{
Reoperation for bleeding in cardiac surgery: surgical bleeding or coagulopathy?
}

\author{
Gökçe Sert, Şule Dede, Aslı Demir, Utku Ünal, Ayşegül Özgök \\ Türkiye Yuksek Ihtisas Research Hospital, Anesthesiology Department, Ankara,Turkey
}

\section{Background and goal of the study}

Reoperation is a risk factor that increases the postoperative mortality and morbidity of cardiac surgery patients. Our aim in this study is to investigate the rates of bleeding-induced reoperation, preoperative and intraoperative risk factors for bleeding, and identify causes of bleeding (surgical or coagulopathic) in our cardiac surgery patient.

\section{Materials and Methods}

After receiving approval from the ethics committee of the hospital, perioperative patient data were collected from hospital electronic system and patient's files between jan-oct2017.We included all cases of adult open heart surgery using cardiopulmonary bypass,except for cases of heart failure surgeries. 1200 patients found, 77 patient have undergone at least one operation within $24 \mathrm{~h}$ due to postoperative bleeding $(\% 6,4)$. Patients' comorbidities, haematological drug use, hypothermia, CPB duration, cardioplegia types, bleeding reasons, postoperative complications and mortality information were investigated.We classified the bleeding as surgical and coagulopathic. Haematoma without ongoing bleeding and/or oozing was defined as coagulopathic bleeding, and spesific bleeding requiring suture or clips was defined as surgical bleeding.

\section{Results and Discussion}

71 were available in the data of patients. 28(39.5\%) patients had surgical bleeding, and $43(60.5 \%)$ patients had coagulopathic bleeding. When the variables that might be a risk factor in terms of type of bleeding in preoperative and intraoperative period were examined, no related factors were found (Table 1 ).

\section{Conclusion}

Our aim was to find factors with significant influence on the risk for reoperation due to bleeding after cardiac surgery and evaluate the consequence of the reoperation. The bleeding was differentiated in coagulopathic and surgical. In total, $39,5 \%$ of the patients had surgical bleeding.Other series in literature, found $56,4 \%$ surgical bleeding ratio(1). It is not possible to distinguish the risk factors because of the low number of patients. However, we can say that the rate of surgical bleeding is lower in our center.

1- Kristensen KL, Rauer LJ, Mortensen PE, Kieldsen BJ. Reoperation for bleeding in cardiac surgery. Interact Cardiovasc Thorac Surg. 2012;14(6):709-13.
Table 1

\begin{tabular}{|c|c|c|c|}
\hline & $\begin{array}{r}\text { Coagulopathic } \\
\text { bleeding }\end{array}$ & Surgical bleeding & $\mathrm{p}$ values \\
\hline Age (Mean \pm std dev) & $61,77 \pm 14,9$ & $58,82 \pm 11$ & 0,32 \\
\hline Gender $(\mathrm{F} / \mathrm{M})(\mathrm{n})$ & $14 / 29$ & $10 / 18$ & 0,49 \\
\hline $\begin{array}{r}\text { Elective/ } \\
\text { emergency(n) }\end{array}$ & $39 / 4$ & $24 / 4$ & 0,38 \\
\hline BMI(Mean \pm std dev) & $25,4 \pm 2,7$ & $26,1 \pm 2,7$ & 0,69 \\
\hline DM \% & 25,6 & 28,6 & 0,49 \\
\hline HT\% & 39,5 & 25 & 0,15 \\
\hline Acetylsalicilic asit\% & 34,9 & 21,4 & 0,17 \\
\hline Previous MI or PCI\% & 16,3 & 10,7 & 0,38 \\
\hline Clopidogrel\% & 7 & 7,1 & 0,66 \\
\hline Coumadin $\%$ & 32,6 & 21,4 & 0,42 \\
\hline $\begin{array}{r}\text { creatinin(Mean } \pm \text { std } \\
\text { dev })\end{array}$ & $0,98 \pm 0,39$ & $0,99 \pm 0,44$ & 0,63 \\
\hline $\begin{array}{r}\text { İntraoperative } \\
\text { tranexamic asit }\end{array}$ & 11,6 & 28,6 & 0,11 \\
\hline $\begin{array}{r}\text { Initial Hct(Mean } \pm \text { std } \\
\text { dev })\end{array}$ & $38,2 \pm 6,5$ & $37,3 \pm 7,6$ & 0,67 \\
\hline $\mathrm{EF}($ Mean \pm std dev) & $50,58 \pm 8,6$ & $49,9 \pm 10$ & 0,67 \\
\hline Nadir hypothermia & $31 \pm 1,1$ & $30,93 \pm 1,8$ & 0,81 \\
\hline $\begin{array}{r}\text { Cardioplegia } \\
\text { type(delnido/plegisol) }\end{array}$ & $12 / 31$ & $9 / 19$ & 0,70 \\
\hline $\begin{array}{l}\text { Cross clamp duration } \\
\text { min (Mean } \pm \text { std dev) }\end{array}$ & $73 \pm 19,9$ & $74 \pm 28$ & 0,79 \\
\hline $\begin{array}{r}\text { CPB duration } \\
\text { min(Mean } \pm \text { std dev })\end{array}$ & $111 \pm 24$ & $111 \pm 44$ & 0,99 \\
\hline $\begin{array}{r}\text { Procedure } \\
\text { (cabg/others) }\end{array}$ & $22 / 21$ & $15 / 13$ & 0,54 \\
\hline $\begin{array}{r}\text { Postoperative } \\
\text { pulmonary } \\
\text { complications\% }\end{array}$ & $25,6 \%$ & $28,6 \%$ & 0,78 \\
\hline $\begin{array}{r}\text { Postoperative } \\
\text { infection } \\
\text { complications\% }\end{array}$ & 16,1 & 32,3 & $\overline{0,43}$ \\
\hline $\begin{array}{r}\text { Postoperative } \\
\text { gastrointestinal } \\
\text { complications\% }\end{array}$ & 0 & 3,6 & 0,21 \\
\hline $\begin{array}{r}\text { Postoperative } \\
\text { cerebrovasculary } \\
\text { complications } \%\end{array}$ & 2,3 & 3,6 & 0,75 \\
\hline 30 days mortality $\%$ & 2,3 & 3,6 & 0,75 \\
\hline
\end{tabular}

